\title{
Detección de plantas asintomáticas de Solanum lycopersicum L. infectadas con Fusarium oxysporum usando espectroscopia de reflectancia VIS
}

\section{Detection of asymptomatic Solanum lycopersicum L. plants infected with Fusarium oxysporum using reflectance VIS spectroscopy}

JUAN CARLOS MARÍN-ORTIZ
LILLIANA MARÍA HOYOS-CARVAJAL
VERÓNICA BOTERO-FERNÁNDEZ ${ }^{2}$

Cultivo de tomate en producción.

Foto: L.M. Hoyos-Carvajal

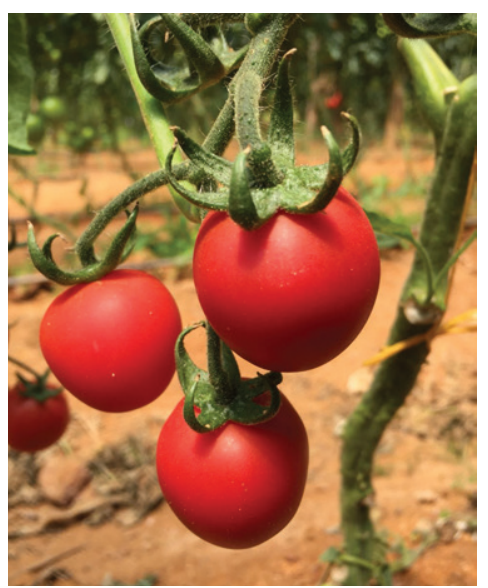

\section{RESUMEN}

Las plantas asintomáticas son reservorios de patógenos, ya que pueden permanecer infectadas la mayor parte de su ciclo de desarrollo, convirtiéndose en fuente de contaminación para el resto del cultivo. El objetivo de este estudio fue evaluar un método de detección y discriminación de dos cepas de Fusarium oxysporum en tomate usando espectroscopia. La enfermedad en las plantas de tomate inoculadas con la cepa aislada de gulupa (F05) fue mayor a la observada en la cepa aislada de tomate (F07), presentando valores de 60,0\% (11 días) y 81,8\% (22 días); la cepa F07 presentó incidencias de 30,0 y 64,3\% en ambas mediciones. La planta infectada con la cepa F05 fue mejor discriminada en el periodo de incubación de la enfermedad en ambos periodos de tiempo en los Análisis de Componentes Principales (PCA) y Análisis Discriminantes Lineales (LDA) realizados con los controles en comparación con la cepa F07. Estos resultados sugieren que la espectroscopia de reflectancia VIS es un método sensible y confiable que puede ser adecuado para el diagnóstico temprano de enfermedades en plantas.

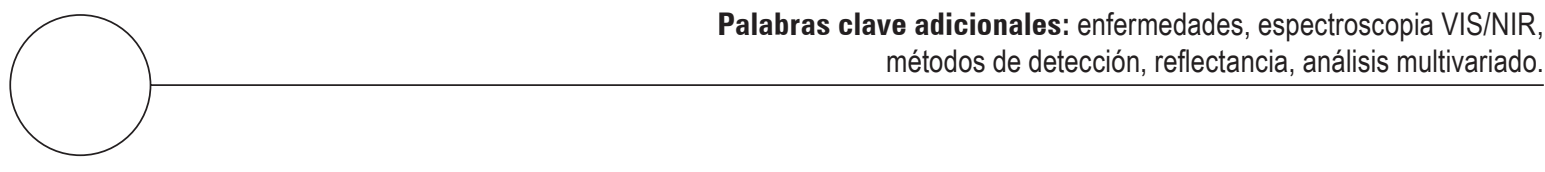

Facultad de Ciencias Agrarias, Universidad Nacional de Colombia, Medellín (Colombia). ORCID Marín-Ortiz, J.C.: 0000-0002-1334-4721; ORCID Hoyos-Carvajal, L.M.: 0000-0003-0980-5035

2 Facultad de Minas, Universidad Nacional de Colombia, Medellín (Colombia). ORCID Botero-Fernández, V.: 0000-0002-6964-239X

3 Autor para correspondencia. juancarlosmo@gmail.com 


\section{ABSTRACT}

Asymptomatic plants are reservoirs of pathogens because they can remain infected most of the development cycle, becoming a source of contamination for the rest of the crop. The objective of this study was to evaluate a method of detection and discrimination of two F. oxysporum isolates on tomato plants using reflectance spectroscopy in the VIS region. The incidence of the fungal isolate from the purple passion fruit plants (F05) was greater than that observed in the strain isolated from the tomato plant (F07), with values of $60.0 \%$ at 11 days and $81.8 \%$ at 22 days; the incidence present in the plants with strain F07 was 30.0\% and $64.3 \%$ in the evaluated period. The F05 strain showed better grouping in both periods of time, both in the Principal Component Analysis and Linear Discriminant Analysis, than the controls, as did the F07 strain. These results suggest that reflectance spectroscopy in the VIS is a sensitive and reliable method that may be suitable for early diagnosis of plant diseases.

Additional key words: diseases, VIS/NIR spectroscopy, detection methods, reflectance, multivariate analysis

Fecha de recepción: 19-09-2017 Aprobado para publicación: 30-05-2018
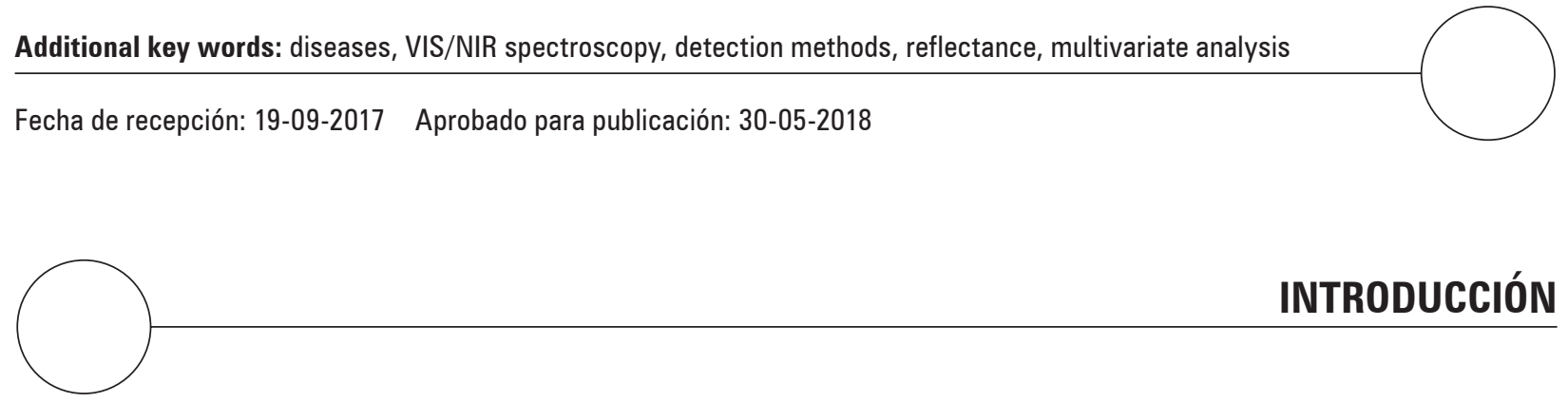

Fusarium oxysporum Schltdl. 1824, es una de las especies morfológicamente más diversas y ampliamente distribuida del género Fusarium (Baayen et al., 2000). Esta especie, se constituye en un complejo, con numerosas formas especiales las cuales son morfológicamente idénticas, solo pueden ser diferenciadas por el tipo de planta(s) atacada(s) (Leslie y Summerell, 2006), con alrededor de 100 formas especiales. Fusarium se caracteriza por producir colonias de rápido crecimiento y morfología muy variable, predominando dos tipos primordiales, una micelial y una pionotal (Garcés et al., 2001). En general, la mayoría de cepas patógenas pueden atacar las plantas de tomate a lo largo de todo su ciclo de desarrollo, produciendo síntomas de marchitamiento, acompañada del amarillamiento parcial de las hojas y el doblamiento de los brotes de la planta enferma. Una característica que permite diagnosticar y diferenciar rápidamente esta enfermedad es la coloración blanquecina, amarillenta o marrón en los haces vasculares; adicionalmente, se puede presentar enanismo en los brotes y disminución del crecimiento general de la planta (Garcés et al., 1999).

Sin embargo, los síntomas exteriores en la planta hospedera no siempre se detectan rápidamente, o esta puede permanecer asintomática durante su ciclo de desarrollo; esto implica que una planta infectada sea en ocasiones difícil de detectar y pueda ser una hospedera asintomática que actúe como reservorio del patógeno, convirtiéndose en una fuente de contaminación para el resto del cultivo (Kolander et al., 2012). En la actualidad se utiliza una gran cantidad de técnicas de detección de enfermedades de plantas, pero éstas presentan varias limitaciones que disminuyen su eficiencia al usarlas para obtener resultados que conduzcan a decisiones de control. Por ejemplo, la detección basada en la observación de síntomas en enfermedades vasculares implica que cuando hay disturbios visibles el hongo u organismo patógeno haya invadido ya el tejido vascular. Los métodos de detección que se basan en técnicas moleculares, microscopía óptica, microscopía electrónica de transmisión, bioensayo para la detección de virus, y la serología, tienen buenos niveles de precisión, pero requieren la destrucción de las muestras, consumen tiempo, son costosos y requieren alta capacitación profesional, por lo que su uso es modesto, implicando imprecisión porque la enfermedad se diagnostica usando tejidos o plantas completas que se destruyen, no siendo óptimas en el sentido estricto de detección temprana y en tiempo de avance de enfermedades de plantas (Sankaran et al., 2011).

Es por estas razones, y a pesar de la disponibilidad de estas técnicas, un sistema de detección temprana de la enfermedad basado en técnicas de espectroscopía puede ayudar a disminuir las pérdidas causadas en los cultivos y evitar una mayor propagación de las enfermedades, evitando por ejemplo la siembra de unidades de plantas portadoras del patógeno, con más rapidez, sensibilidad, selectividad y sin demandar la destrucción de muestras requeridas para el análisis (Chaerle 
y Van der Straeten, 2000). En la última década se han realizado muchos estudios en los cuales fue aplicada la espectroscopía y particularmente, el uso de radiación VIS en la detección de enfermedades en plantas sintomáticas (Sankaran et al., 2011; Abu-Khalaf y Salman, 2014; Szuvandzsiev et al., 2014); sin embargo, aquellos enfocados en la detección de enfermedades en plantas asintomáticas o estudios enfocados a enfermedades que pueden "enmascarar" los síntomas usan la espectrometría de reflectancia en proporciones relativamente bajas. Por lo anterior, el objetivo de este estudio fue evaluar un método de detección y discriminación de la respuesta espectral debida a cambios fisiológicos en plantas de tomate asintomáticas infectadas con dos cepas de F. oxysporum usando espectroscopía de reflectancia en la región visible (VIS).

\section{MATERIALES Y METODOS}

\section{Material vegetal}

En este estudio se usó la variedad de tomate, tipo Chonto Santa $\mathrm{Cruz}^{\circledast}$, resistente a F. oxysporum. Las semillas fueron plantadas en bandejas de germinación de 76 pozos con turba estéril como sustrato y se mantuvieron en una cámara de siembra con temperatura de $26 \pm 1^{\circ} \mathrm{C}$, humedad relativa de $60 \pm 10 \%$ y fotoperiodo de $12 \mathrm{~h}$ con luz blanca fluorescente, con una intensidad entre 40 a $80 \mathrm{~mol}^{-2} \mathrm{~s}^{-1}$ durante el tiempo de los experimentos. Las plantas se regaron diariamente y se fertilizaron una vez por semana con una solución hidropónica que contenía (mili-equivalentes): $\mathrm{NO}_{3}$ $(12,0), \mathrm{PO}_{4}(1,0), \mathrm{K}(1,7), \mathrm{Mg}(1,5), \mathrm{Ca}(2,8)$ y S $(0,5)$, y en micro-equivalentes, $\mathrm{Fe}(70,0), \mathrm{Mn}(18,0), \mathrm{Zn}(7,7)$, $\mathrm{Cu}(1,5), \mathrm{B}(27,5)$ y Mo $(0,5)$. A las 4 semanas después de germinadas (sdg) se realizó el procedimiento de inoculación y las plantas se transfirieron a recipientes de $90 \mathrm{~cm}^{3}$ que contenían el mismo sustrato que el usado en plantulación. Las plantas inoculadas se mantuvieron en las mismas condiciones de cámara de siembra durante el resto del experimento, aumentando las fertilizaciones a dos veces por semana con las mismas concentraciones en macro y micronutrientes (Lafontaine y Benhamou, 2010).

\section{Preparación de aislamientos de $F$. oxysporum}

Se usaron dos cepas de F. oxysporum aisladas de hospederos diferentes; la cepa F05 fue aislada de Passiflora edulis (gulupa) (cepa A54; Ortiz y Hoyos-Carvajal,
2016) y la cepa F07 fue aislada de plantas de Solanum lycopersicum (tomate). Ambas fueron mantenidas en cajas de Petri con agar papa dextrosa (Merck ${ }^{\circledR}$ ) PDA + $50 \mathrm{mg} \mathrm{kg}^{-1}$ de sulfato de estreptomicina (SE). Multiplicándose según las necesidades.

\section{Inoculación de plantas de tomate antes del trasplante definitivo}

Para este experimento se usaron 12 plantas de $4 \mathrm{sdg}$ siguiendo la metodología de inoculación descrita por Ortiz y Hoyos-Carvajal (2016) y 12 plantas control. Se utilizó la concentración $1 \cdot 10^{6}$ esporas $/ \mathrm{mL}$ por cada aislamiento de Fusarium de 10 d de cultivo. Cuidadosamente las raíces de las plántulas fueron lavadas con agua de grifo hasta retirar los restos de turba. Luego de la limpieza se hacen heridas (cortes) en las raíces terciarias de todas las plantas que se infectaron con tijeras esterilizadas y se sumergen solo las raíces en $100 \mathrm{~mL}$ de la solución de esporas durante $10 \mathrm{~min}$. Las plántulas inoculadas y controles fueron trasplantados a los vasos con turba estéril de $5 \mathrm{~cm}^{3}$. En las plantas control se realizó el mismo procedimiento de las plantas inoculadas, pero solo con agua destilada. Para llevar a cabo los postulados de Koch se realizaron muestreos destructivos a los 11 y 22 días pos-inoculación (dpi).

\section{Prueba de infectividad}

Para confirmar la infección de las plantas se realizaron dos muestreos destructivos, en los estados 1.10 (11 dpi) y el estado 2.20 (22 dpi), según la escala $\mathrm{BBCH}$ para las mono y dicotiledóneas (Feller et al., 1995). Se realizó la prueba de infectividad mediante la técnica de indexación en medio PDA + SE a $50 \mathrm{mg} \mathrm{kg}^{-1}$ (Ortiz y Hoyos-Carvajal, 2016). A los 5 d después de sembrar los segmentos indexados de tallo en el medio usado se observó la presencia y crecimiento del patógeno.

\section{Espectroscopia}

Para la adquisición de espectros de reflectancia VIS se utilizó un espectroscopio portátil USB2000+ (Ocean Optics, Largo, FL) con una fuente de luz halógena de tungsteno HL-2000-HP (rango de longitud de onda de 360-2.400 nm), un estándar de reflectancia difusa modelo WS-1 (reflectividad $>98 \%$ en el rango de 250 $1.500 \mathrm{~nm}$ ) y una sonda de reflectancia grado premium de $600 \mu \mathrm{m}$ OR600-7-VID-125F (Ocean Optics, Largo, FL, USA). Las mediciones se realizaron en los estados 
fenológicos 1.10 y 2.20 de la planta en las hojas 2 y 3 con la fibra óptica en la cara adaxial obteniéndose cinco espectros por cada hoja. El tiempo de integración utilizado en esta investigación fue de $1 \mathrm{~ms}$, un promedio de 10 lecturas por medición y "tiempo de intervalo" de $2000 \mu \mathrm{s}$.

\section{Análisis de datos}

La discriminación y comparación de los efectos causados por ambas cepas se llevó a cabo en hojas en el mismo estadio de crecimiento y desarrollo, sólo usándose hojas desarrolladas y de la misma edad (hoja dos de cada planta). Los análisis realizados en los datos de las plantas 22 dpi tienen un mayor muestreo. Intencionalmente, se usó mayor cantidad de plantas, tratando de prever una mortalidad natural que al fin del experimento fue muy baja, por lo que no todas las muestras contienen el mismo número de espectros.

Inicialmente, se realizó una selección de los espectros en la cual se retiraron algunos con ruido (deformados y/o con error de lectura). Estos espectros que mostraron patrones muy diferentes se confirmaron con análisis de los "valores atípicos" identificados en un Análisis de Componentes Principales (PCA) sin tratamiento previo de datos. Después de la eliminación de los espectros con ruido y tratamientos previos, se aplicó la combinación de las transformaciones Corrección Dispersión Multiplicativa (MSC) más la "segunda derivada" como mejor pre-tratamiento, lo que permite una buena agrupación de las plantas en los tratamientos realizados (Isaksson y Naes, 1998). Luego del pre-tratamiento de datos se realizaron los PCA y LDA enfocados a evaluar la posible separación entre plantas infectadas y las de control en diferentes tiempos pos-infección (Fig. 1).

Los espectros de las plantas infectadas con cada cepa de F. oxysporum (F05 y F07) se analizaron separados con los controles (no se realizó tratamiento combinando las cepas) y en los dos tiempos pos-infección. El conjunto total de datos tomados de las hojas 2 y 4 de las plantas infectadas y controles se compone de 156 espectros (promedios de cinco espectros tomados por hoja). En este análisis solo se tomaron los 78 espectros correspondientes a la hoja 2 para evitar ruido relacionado con la diferencia en el tiempo de desarrollo de ambas hojas, los cuales fueron medidos desde 10 plantas control, 10 infectadas con la cepa F05 y 10 infectadas con la cepa F07 en el día 11, y 13 controles,
20 infectadas con la cepa F05 y 17 infectadas con la cepa F07 en el día 22. Después de la eliminación de los espectros con ruido y "atípicos" se usaron 64 espectros en total.

\section{RESULTADOS}

\section{Confirmación de la infección}

Se evaluó la incidencia de la enfermedad en plantas de tomate inoculadas con F. oxysporum en plantas de tomate con base en el porcentaje de individuos infectados en relación con el total de las plantas inoculadas. La incidencia de la cepa F05, que fue aislada de una planta de gulupa, fue mayor a la observada en la cepa F07 (aislada de una planta de tomate), presentando valores del $60,0 \%$ a los $11 \mathrm{~d}$ y $81,8 \%$ a los $22 \mathrm{~d}$. La cepa F07 presentó incidencias de 30,0\% y $64,3 \%$ en los tiempos evaluados (11 y $22 \mathrm{~d}$, respectivamente).

\section{Análisis de datos espectrales}

El Análisis de Componentes Principales (PCA) obtenidos de los espectros de las hojas de tomate infectadas con las cepas de F. oxysporum, F05 y F07, se agruparon según la cepa y tiempo pos-infección. La cepa F05 presentó mejor agrupamiento en los dos periodos de tiempo pos-inoculación con respecto a los controles (Fig. 2A y 2A') y la cepa F07 (Fig. 2C y 2C'). Sin embargo, la cepa F07 no presentó un agrupamiento claro a los 11 dpi y mejoró considerablemente para a los 22 dpi (Fig. 2B y 2B'). Es importante resaltar que estos resultados son coherentes con los obtenidos en los muestreos destructivos para confirmación de la infección, en la cual se dio mayor porcentaje de infección para la cepa F05 en ambos periodos de tiempo, y la cepa $\mathrm{F} 07$ tuvo un porcentaje muy bajo de infección en el día 11 (30\%).

El PCA realizado con la cepa F05 junto con sus controles (Fig. 2A y 2A') se emplearon 41 espectros, los componentes principales CP1 y CP2 explican el 66\% $(\mathrm{CP} 1=60 \%, \mathrm{CP} 2=6 \%)$ y $76 \%(\mathrm{CP} 1=70 \%, \mathrm{CP} 2=6 \%)$ de la variabilidad total de los datos en los 11 y $22 \mathrm{dpi}$, respectivamente. Adicionalmente, se usaron 43 espectros para los PCA realizados con la cepa F07 más los respectivos controles (Fig. 2B y 2B'), en los cuales los CP1 y CP2 solo explican el 29\% (CP1 $=18 \%$, $\mathrm{CP} 2=11 \%)$ y $64 \%(\mathrm{CP} 1=41 \%, \mathrm{CP} 2=23 \%)$ en los 11 y 22 dpi, respectivamente. Finalmente, se usaron 22 


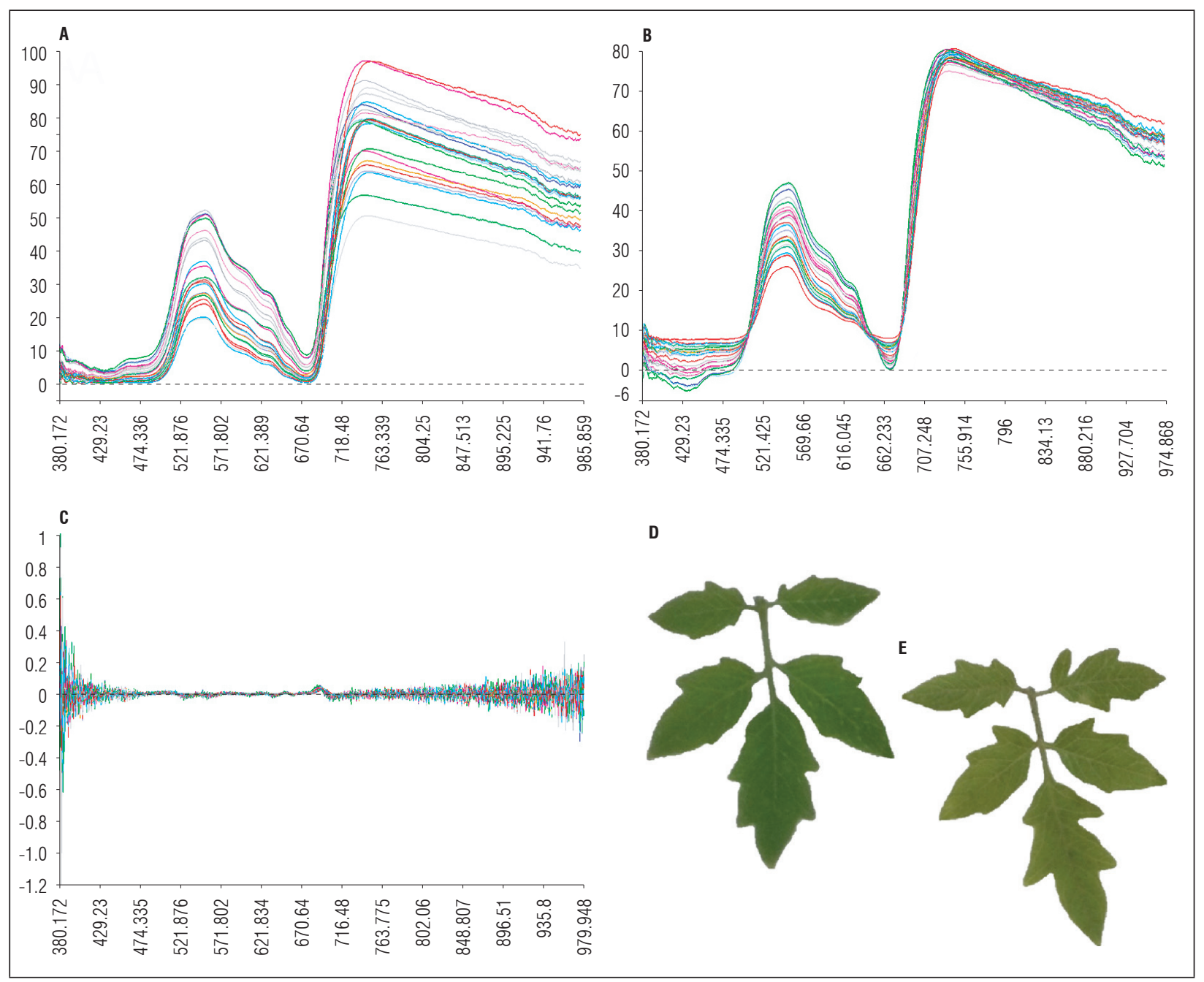

Figura 1. Ejemplo de datos espectrales usados para los análisis mostrando la diferencia entre los pre-tratamientos aplicados y hojas monitoreadas del día 22 después de inoculación: A. Perfiles espectrales sin transformación; B. Corrección de Dispersión Multiplicativa (MSC); C. MSC + Segunda derivada; D. Hoja de planta control; E. Hoja inoculada.

espectros de hojas infectadas con la cepa FO5 y 22 de la cepa FO7 para evaluar si el método pude separar plantas infectadas con aislados diferentes de la misma especie (Fig. 2C y 2C'); en estos PCA, los CP1 y CP2 explicaron el $66 \%$ (CP1 $=61 \%$, CP2 $=5 \%)$ y $68 \%$ (CP1 $=54 \%, C P 2=14 \%$ ) de la variabilidad total de los datos en los días 11 y 22 dpi, respectivamente.

El LDA permitió mejorar la separación de los respectivos tratamientos. Los LDA obtenidos a partir de los datos espectrales de la cepa F05 (Fig. 3A y 3A') alcanzaron el $100 \%$ de precisión obtenidos con la aplicación de cinco componentes principales que predicen las muestras de acuerdo con el tratamiento al cual pertenecen. Por el contrario, los LDA obtenidos de los espectros de las hojas infectadas con la cepa F07 obtuvieron una precisión de solo $58,82 \%$ a los 11 d (Fig. 3B) y un aumento al 80,77\% en el día 22 (Fig. 3B') con la misma aplicación de cinco componentes principales para la predicción de muestras.

En las matrices de confusión se puede confirmar que los diferentes modelos clasificaron correctamente todas las plantas infectadas con la cepa F05 con respecto a las inoculadas con la cepa F07 y controles en ambos días de medición (precisión el 100\%), mientras que para la cepa $\mathrm{F} 07$ el $50 \%$ se clasificó como control el día 11 y el $44 \%$ el día 22 (Tab. 1), lo que denota una clara dificultad del modelo en la discriminación entre las plantas no inoculadas y plantas inoculadas con la cepa F07, las posibles razones serán abordadas en la discusión. 


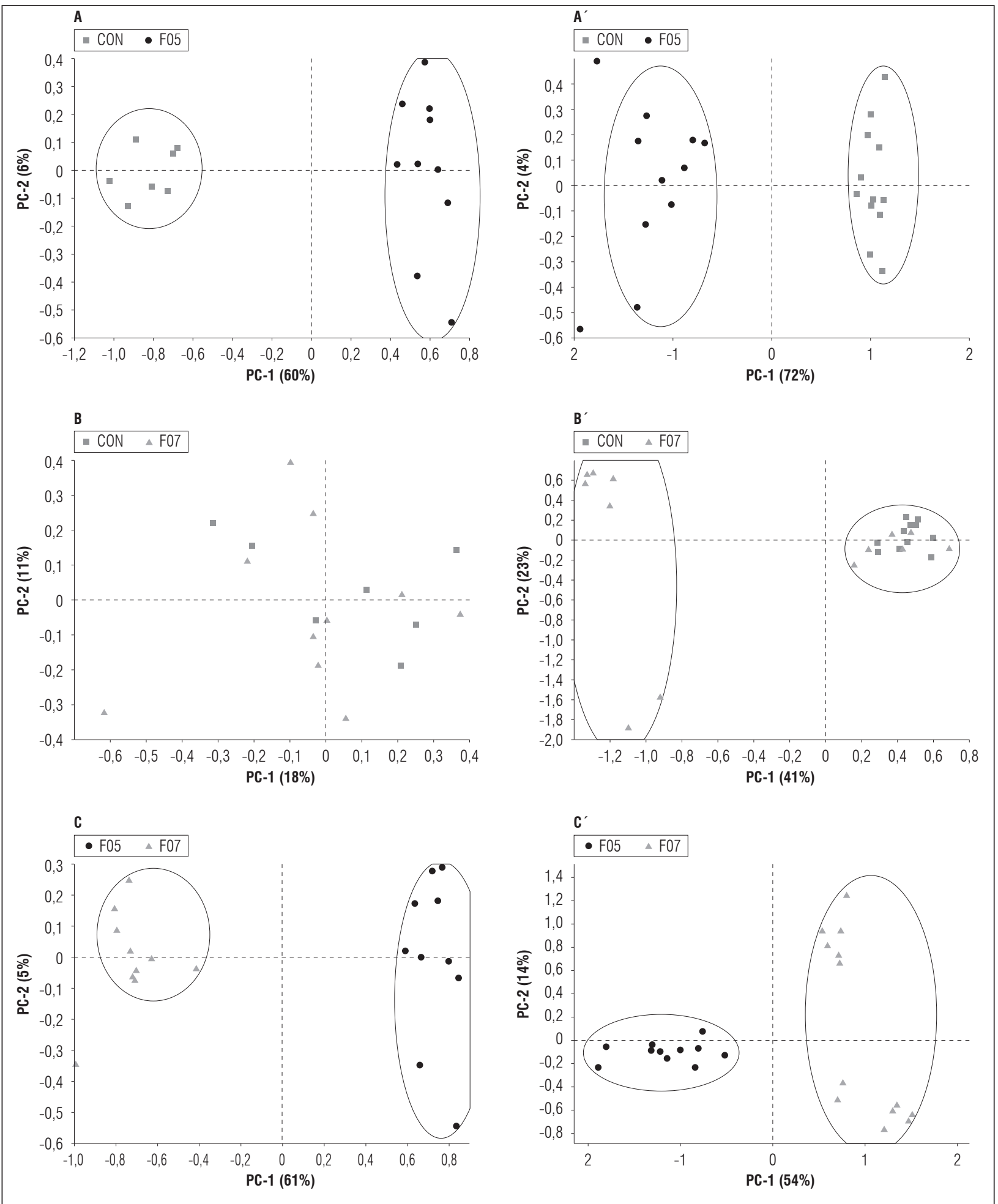

Figura 2. Análisis de Componentes Principales de espectros de tomate de mesa infectadas con dos cepas de $F$. oxysporum y dos tiempos después de la infección (dpi). A. Cepa F05 a los 11 dpi; A'. Cepa F05 a los 22 dpi; B. Cepa F07 a los 11 dpi; B'. Cepa F07 a los 22; C. Cepas F05 y F07 a los 11 dpi; C'. Cepas F05 y F07 a los 22 dpi. 


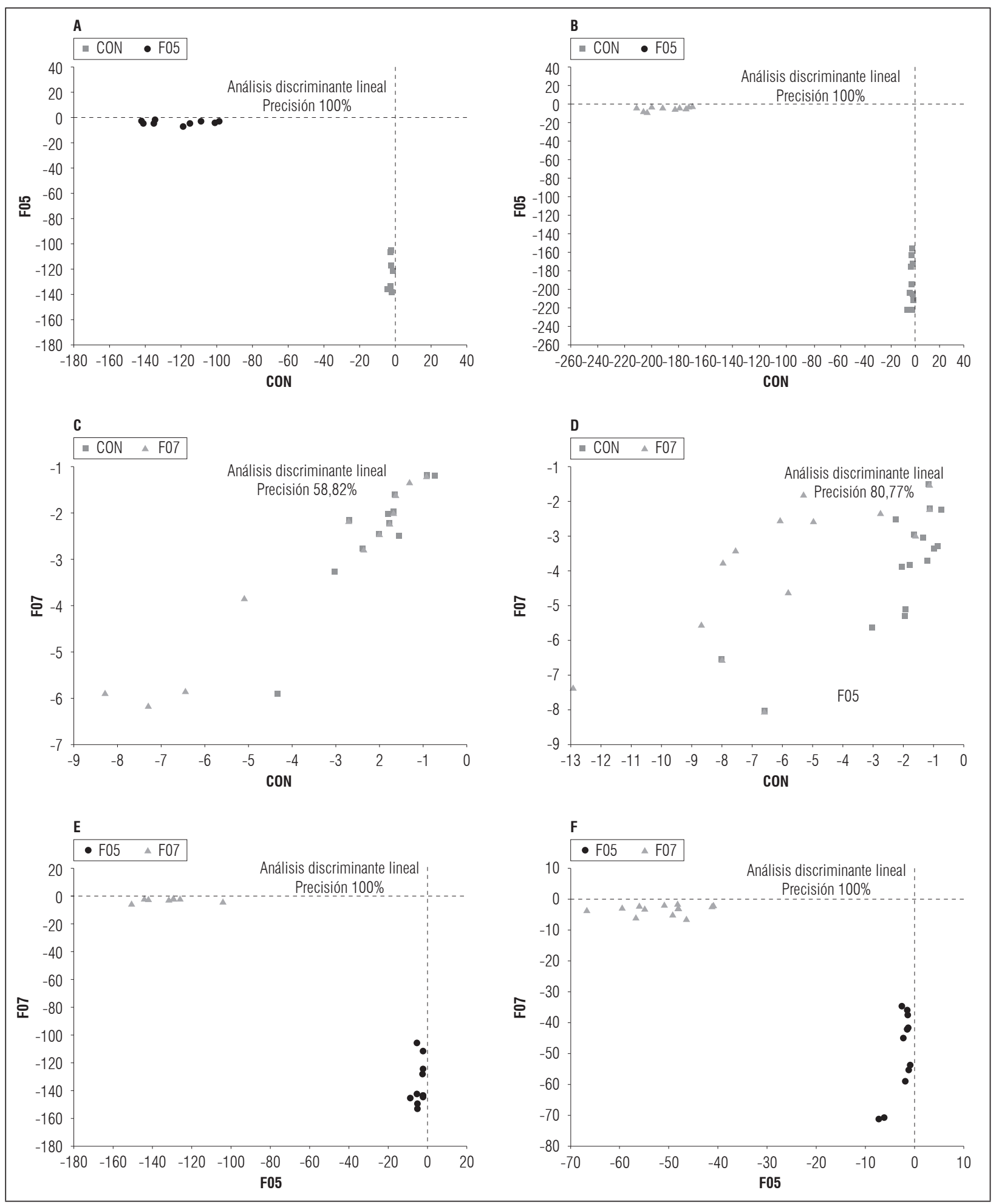

Figura 3. Análisis discriminantes lineales de espectros de tomate de mesa infectadas con dos cepas de $F$. oxysporum y dos tiempos después de la infección (dpi). A. Cepa F05 a los 11 dpi; A'. Cepa F05 a los 22 dpi; B. Cepa F07 a los 11 dpi; B'. Cepa F07 a los 22; C. Cepas F05 y F07 a los 11 dpi; C'. Cepas F05 y F07 a los 22 dpi. 


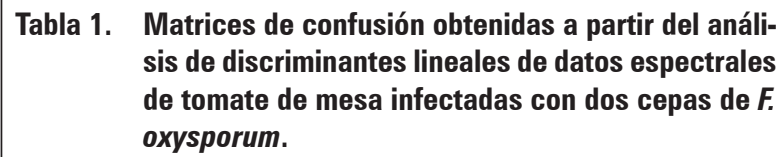

\begin{tabular}{|c|c|c|c|c|}
\hline Pronóstico & \multicolumn{2}{|c|}{ Día 11 } & \multicolumn{2}{c|}{ Día 22 } \\
\hline & CON & F05 & CON & F05 \\
\hline CON & 7 & 0 & 13 & 0 \\
\hline F05 & 0 & 10 & 0 & 11 \\
\hline & CON & F07 & CON & F07 \\
\hline CON & 5 & 5 & 12 & 4 \\
\hline F07 & 2 & 5 & 1 & 9 \\
\hline & F05 & F07 & F05 & F07 \\
\hline F05 y & 10 & 0 & 11 & 0 \\
\hline F07 & 0 & 10 & 0 & 13 \\
\hline
\end{tabular}

\section{DISCUSIÓN}

No se observaron los síntomas típicos del marchitamiento por $F$. oxysporum en el tiempo del ensayo, como epinastia, marchitez no uniforme de hojas o ramas y decoloración vascular extendida por encima de la línea del suelo. Aunque las raíces no fueron observadas en el trascurso del experimento, el muestreo destructivo permitió confirmar la presencia de ambas cepas (F05 y F07) en los periodos de tiempo evaluados (11 y 22 d después de inoculación) mediante identificación morfológica clásica. Se encontró mayor incidencia de la cepa F05 en las plantas de tomate en comparación con la cepa F07, ya que fueron las plantas con mayor incidencia en ambos muestreos destructivos, lo cual fue confirmado en los análisis PCA y LDA realizados con los datos espectrales. Se puede considerar que la cepa F05 tiene una alta capacidad para infectar las plantas de tomate, sin embargo, debido a la resistencia de las variedades de tomate (Jones y Crill, 1974; Rivard y Louws, 2011; Morid et al., 2012), se imposibilita la aparición de los síntomas.

Las hojas enfermas producen cambios fisiológicos significativos antes de la colonización de las hifas de F. oxysporum, por lo que es posible la clasificación de una planta como "enferma" con criterios basados en fluoresencia y/o espectroscopia una o dos semanas antes de que aparezcan los síntomas evidentes. Es importante destacar la concordancia de nuestros resultados con los obtenidos en el estudio de Lorenzini et al. (1997), en el cual encontraron que los parámetros de fluorescencia de la clorofila de las primeras hojas asintomáticas fueron alterados por la infección de F. oxysporum hasta el día 14 después de la infección, debido posiblemente a una disminución de la fluorescencia variable y confirmado por la actividad alterada del fotosistema II. También es importante destacar las diferencias obtenidas entre las dos cepas de F. oxysporum usadas para infectar las plantas de tomate, debido a que la cascada de eventos que ocurren en las células inoculadas con microconidias de aislados con diferente nivel de patogenicidad cambia la relación específica planta-patógeno; desafortunadamente, los mecanismos implicados son complejos y aún no están claramente definidos (Olivain et al., 2003).

Los resultados encontrados en los espectros de reflectancia concuerdan con los muestreos destructivos. Además, se observó una dinámica característica de los espectros de reflectancia medidos en el rango espectral para los días 11 y 22 dpi en las cepas estudiadas, F05 y F07. Esta dinámica se puede observar claramente cuando se comparan las mayores incidencias obtenidas de los muestreos destructivos en las plantas infectadas con los análisis de los datos espectrales (PCA y LDA) de la cepa F05, en los cuales las plantas se agrupan claramente y puede discriminarse de las plantas sin enfermedad y las infectadas en los dos días de medición. Por el contrario, la enfermedad tiene una baja incidencia en las plantas infectadas con la cepa F07 (particularmente en el día 11) y en los análisis de los espectros de reflectancia solo es posible agrupar y discriminar los individuos infectados al día 22 , cuando la incidencia sube al 64,3\%.

Las alteraciones fisiológicas o bioquímicas entre las plantas infectadas y sanas pueden ser causadas por la composición química del parásito o una respuesta desde la planta hospedera, implicando cambios en la ubicación de nutrientes y energía, hasta producción de metabolitos secundarios (Ramachandra y Ravishankarb, 2002), además de posibles respuestas causadas por estrés en las plantas sugerido por cambios en segmentos del infrarrojo cercano (Ferri et al, 2004; Gitelson et al., 2005; Ustina et al., 2009). En ese sentido, los resultados de este estudio son contrarios a los obtenidos por (Spinelli et al., 2006), los cuales usaron técnicas basadas en espectroscopia de reflectancia en el NIR para detectar el "fuego bacteriano" en plantas de peral asintomáticas, sin lograr discriminar las plantas infectadas de plantas sanas. Los autores discuten que sus resultados posiblemente fueron debido a la pequeña escala de medición en la hoja $\left(2 \mathrm{~mm}^{2}\right)$, lo cual pudo ser contrarrestado en nuestro estudio ya que se tomaron cinco mediciones por hoja en las 
cuales cada espectro medido es el resultado promedio de 100 mediciones.

Adicionalmente, es importante destacar que las respuestas fisiológicas e histológicas en plantas hospederas asintomáticas y en etapas tempranas de la infección fúngica que están relacionadas con cambios en las propiedades ópticas de las hojas en las plantas aún son desconocidas en su mayor parte. Además, la mayoría de estudios enfocados a predecir la química foliar son basados en análisis de tejido seco, lo que imposibilita el muestreo de la misma hoja en el tiempo (Fourty et al., 1996; Couture et al., 2013), lo cual ofrece grandes ventajas para la caracterización de respuestas de las plantas a patógenos usando muestras frescas $y / 0$ muestreo in situ como lo abordaron en Franke y Menz, (2007), Naidua et al. (2008), Mahlein et al. (2012), en los cuales se usaron técnicas de teledetección en cultivos de trigo para detectar infección con oídio y roya de la hoja en diferentes periodos de tiempo del cultivo. Estos resultados concuerdan con la base teórica propuesta por Zhang et al. (2003), en la cual se sugiere que la magnitud de onda variará típicamente en diferentes longitudes, aumentando la reflectancia en el VIS y disminuyendo en el NIR (750$1100 \mathrm{~nm}$ ) en las plantas infectadas con patógenos y sometidos a diferentes tipos de estrés.

\section{CONCLUSIONES}

Se probó un método para detectar y discriminar la respuesta espectral debida a cambios en la fisiología de las plantas de tomate asintomáticas infectadas con dos cepas de F. oxysporum usando espectroscopía de reflectancia. Los resultados sugieren que las plantas de tomate pueden mantener niveles relativamente altos de infección sin mostrar síntomas visibles y que es posible detectar y discriminar infecciones asintomáticas de F. oxysporum en plantas usando espectroscopia de reflectancia con radiación en el VIS/NIR. Las cepas de F. oxysporum usadas en este estudio causaron diferencias claras en los espectros de reflectancia en las plantas infectadas y las no infectadas, además de permitir una buena discriminación entre las plantas infectadas con ambas cepas.

Los resultados obtenidos indican que la espectroscopia VIS/NIR es promisoria para la detección de hongos vasculares, incluso en plantas asintomáticas. Adicionalmente, la aplicación de técnicas multivariadas como PCA y LDA a datos espectrales en el VIS/ NIR de muestras frescas inoculadas con diferentes cepas de F. oxysporum ha mostrado ser un método eficiente para la detección y discriminación de infecciones fúngicas.

Aunque son varias las técnicas espectroscópicas y de imágenes que se han usado para la detección de enfermedades en plantas sintomáticas, es muy baja la cantidad de estudios especializados en la detección de enfermedades en plantas asintomáticas usando técnicas no destructivas como la espectroscopía de reflectancia. En este sentido, los resultados de este estudio son valiosos como contribución en esta área del conocimiento. Adicionalmente, la correspondencia entre la medición de la incidencia de la enfermedad y los cambios encontrados por la técnica de espectroscopía de reflectancia en plantas asintomáticas sugiere que es un método sensible y confiable que puede ser adecuado para el diagnóstico temprano de enfermedades en plantas. Sin embargo, los mecanismos fisiológicos y anatómicos que influyen en la expresión de síntomas de la planta debido a las infecciones fúngicas aun parecen muy complejos y no están claramente definidos, particularmente aquellos mecanismos presentes en etapas tempranas de la infección, lo cual invita a desarrollar nuevos proyectos de investigación que profundicen y aclaren esta área del conocimiento de gran importancia en enfermedades tanto vasculares como sistémicas.

\section{AGRADECIMIENTOS}

Los autores desean agradecer a todo el personal del Laboratorio de Sanidad Vegetal de la Universidad Nacional de Colombia, sede Medellín. Adicionalmente, agradecemos al Dr. Lucio Flavio de Alencar Figueiredo del Departamento de Botánica de la Universidad de Brasilia por sus valiosos comentarios.

Conflicto de intereses: el manuscrito fue preparado y revisado con la participación de los autores, quienes declaran no tener algún conflicto de interés que coloquen en riesgo la validez de los resultados aquí presentados.

\section{REFERENCIAS BIBLIOGRÁFICAS}

Abu-Khalaf, N. y M. Salman. 2014. Visible/Near infrared (VIS/NIR) spectroscopy and multivariate data analysis (MVDA) for identification and quantification of olive leaf spot (OLS) disease. Palest. Tech. Univ. Res. J. 2(1), 1-8. 
Baayen, R., P.K. O’Donnell, P.J. Bonants, E. Cigelnik, L. Kroon, E.J. Roebroeck y C. Waalwijk. 2000. Gene genealogies and AFLP analyses in the Fusarium oxysporum complex identify monophyletic and nonmonophyletic formae speciales causing wilt and rot disease. Phytopathology 90, 891-900. Doi: 10.1094/ PHYTO.2000.90.8.891

Chaerle, L. y D. Van der Straeten. 2000. Imaging techniques and the early detection of plant stress. Trends Plant Sci. 5, 495-501. Doi: 10.1016/S1360-1385(00)01781-7

Couture, J.J., S.P. Serbin y P. Townsend. 2013. Spectroscopic sensitivity of real-time, rapidly induced phytochemical change in response to damage. New Phytol. 198(1), 311-9. Doi: 10.1111/nph.12159

Feller, C., H. Bleiholder, L. Buhr, H. Hack, M. Hess, R. Klose, U. Meier, R. Stauss, T. van den Boom y E. Weber. 1995. Phänologische Entwicklungsstadien von Gemüsepflanzen: Fruchtgemüse und Hülsenfrüchte. Nachrichtenblatt des Deutschen Pflanzenschutzdienstes $356,217-232$

Ferri, C., R. Formaggio y M. Schiavinato. 2004. Narrow band spectral indexes for chlorophyll determination in soybean canopies [Glycine max (L.) Merril]. Braz. J. Plant Physiol. 16(3), 131-136. Doi: 10.1590/ S1677-04202004000300002

Fourty, T., F. Baret, S. Jacquemoud, G. Schmuck y J. Verdebout. 1996. Leaf optical properties with explicit description of its biochemical composition: direct and inverse problems. Remote Sens. Environ. 56, 104-117. Doi: 10.1016/0034-4257(95)00234-0

Franke, J. y G. Menz. 2007. Multi-temporal wheat disease detection by multi-spectral remote sensing. Precision Agric. 8(3), 161-172. Doi: 10.1007/s11119-007-9036-y

Garcés, E., A. Orozco y A.C. Zapata. 1999. Fitopatología en flores. Acta Biol Colomb. 4(2), 5-26.

Garcés, E., G.R. Bautista y H. Valencia. 2001. Fusarium oxysporum el hongo que nos falta conocer. Acta Biol. Colomb. 6(1), 7-25.

Gitelson, A., A. Viña, V. Ciganda, D. Rundquist y T.J. Arkebauer. 2005. Remote estimation of canopy chlorophyll content in crops. Geophys. Res. Lett. 32. Doi: 10.1029/2005GL022688

Isaksson, T. y T. Naes. 1988. The effect of multiplicative scatter correction (MSC) and linearity improvement in NIR spectroscopy. Appl. Spectrosc. 42(7), 12731284. Doi: $10.1366 / 0003702884429869$

Jones, J. y P. Crill. 1974. Susceptibility of "resistant" tomato cultivars to fusarium wilt. Phytopathology 64, 15071510. Doi: 10.1094/Phyto-64-1507

Kolander, T., M.J.C. Bienapfl, J.E. Kurle y D.K. Malvick. 2012. Symptomatic and asymptomatic host range of Fusarium virguliforme, the causal agent of soybean sudden death syndrome. Plant Dis. 96(8), 1148-1153. Doi: 10.1094/PDIS-08-11-0685-RE
Lafontaine, P. y N. Benhamou. 2010. Chitosan treatment: an emerging strategy for enhancing resistance of greenhouse tomato plants to infection by Fusarium oxysporum f.sp. radicis-lycopersici. Biocontrol Sci. Technol. 6(1), 111-124. Doi: 10.1080/09583159650039575

Leslie, J.F. y B.A. Summerell. 2006. The Fusarium laboratory manual. Blackwell Publishing, Ames, IA, USA. Doi: 10.1002/9780470278376

Lorenzini, G., L. Guidi, C. Nali. C. Ciompi y G.F. Soldatini. 1997. Photosynthetic response of tomato plants to vascular wilt diseases. Plant Sci. 124(2), 143-152. Doi: 10.1016/S0168-9452(97)04600-1

Mahlein, A.K., U. Steiner, C. Hillnhütter, H. Dehne y E.C. Oerke. 2012. Hyperspectral imaging for small-scale analysis of symptoms caused by different sugar beet diseases. Plant Methods 8(3), PMC3274483. Doi: 10.1186/1746-4811-8-3

Morid, B., S. Hajmansoor y N. Kakvan. 2012. Screening of resistance genes to fusarium root rot and fusarium wilt diseases in tomato (Lycopersicon esculentum) cultivars using RAPD and CAPs markers. Eur. J. Exp. Biol. 2(4), 931-939.

Naidua, R.A., E.M. Perryb, F.J. Pierceb y T. Mekuriaa. 2008. The potential of spectral reflectance technique for the detection of grapevine leafroll-associated virus-3 in two red-berried wine grape cultivars. Comput. Electron. Agr. 66, 38-45. Doi: 10.1016/j. compag.2008.11.007

Olivain, C., S. Trouvelot, M.N. Binet, C. Cordier, A. Pugin y C. Alabouvette. 2003. Colonization of flax roots and early physiological responses of flax cells inoculated with pathogenic and nonpathogenic strains of Fusarium oxysporum. Appl. Environ. Microbiol. 69(9), 54535462. Doi: 10.1128/AEM.69.9.5453-5462.2003

Ortiz, E. y L. Hoyos-Carvajal. 2016. Standard methods for inoculations of F. oxysporum and F. solani in Passiflora. Afr. J. Agric. Res. 11(17), 1569-1575. Doi: 10.5897/ AJAR2015.10448

Ramachandra, R. y G.R. Ravishankarb. 2002. Plant cell cultures: chemical factories of secondary metabolites. Biotechnol. Adv. 20, 101-153. Doi: 10.1016/ S0734-9750(02)00007-1

Rivard, C. y F. Louws. 2011. Tomato grafting for disease resistance and increased productivity. Agricultural Innovations. Sustainable Agriculture Research and Education (SARE) program. Fact Sheets 12AGI2011. En: http://www.sare.org/factsheet/12AGI2011 consulta: febrero de 2018.

Sankaran, S., A. Mishra, J. Mari y R. Ehsani. 2011. Visible-near infrared spectroscopy for detection of Huanglongbing in citrus orchards. Comput. Electron. Agr. 77, 127-134. Doi: 10.1016/j.compag.2011.03.004

Spinelli, F., M. Noferini y G. Costa. 2006. Near infrared spectroscopy (NIRs): Perspective of fire blight detection in 
asymptomatic plant material. Acta Hortic. 704, 87-90. Doi. 10.17660/ActaHortic.2006.704.9

Szuvandzsiev, P., L. Helyes, A. Lugasi, C. Szántó, P. Baranowski y Z. Pék. 2014. Estimation of antioxidant components of tomato using VIS-NIR reflectance data by handheld portable spectrometer. Int. Agroph. 28(4), 521-527. Doi: 10.2478/intag-2014-0042

Ustina, S.L., A.A. Gitelsonb, S. Jacquemoudc, M. Schaepmand, G. Asnere, J.A. Gamonf y P. Zarco-Tejadag.
2009. Retrieval of foliar information about plant pigment systems from high resolution spectroscopy. Remote Sens. Environ. 113(1), 67-77. Doi: 10.1016/j. rse.2008.10.019

Zhang, M., Z. Oin, X. Liu y S.L. Ustin. 2003. Detection of stress in tomatoes induced by late blight disease in California, USA, using hyperspectral remote sensing. Int. J. Appl. Earth Observ. Geoinf. 4, 295-310. Doi: 10.1016/S0303-2434(03)00008-4 